Supporting Information

\title{
Interface and Doping Effect on the Electrochemical Property of Graphene/LiFePO4
}

Huayu Wang $^{a}$, Naiqin Zhao ${ }^{a, b}$, Chunsheng Shi ${ }^{a}$, Chunnian He ${ }^{a}$, Jiajun Li ${ }^{a}$, Enzuo $\operatorname{Liu}^{a, b *}$

${ }^{\mathrm{a}}$ Key Laboratory of Composite and Functional Materials, School of Materials Science and Engineering, Tianjin University, Tianjin 300072, China

${ }^{\mathrm{b}}$ Collaborative Innovation Center of Chemical Science and Engineering, Tianjin University, Tianjin 300072, China

\section{Corresponding Author}

*Enzuo Liu. E-mail: ezliu@tju.edu.cn 
Table S1. The lattice mismatch (\%) between graphene and $\mathrm{LiFePO}_{4}(\mathrm{LFPO})$ surface and the bulk potentials (V) of LFPO and $\mathrm{LiMnPO}_{4}$. Reference values are listed for comparison.

\begin{tabular}{|c|c|c|c|c|c|}
\hline & \multicolumn{2}{|c|}{ Lattice mismatch } & \multirow{2}{*}{$\mathrm{GGA}+\mathrm{U}$} & \multirow{2}{*}{$\begin{array}{l}\text { GGA+U } \\
\text { Ref. [1] }\end{array}$} & \multirow{2}{*}{$\begin{array}{c}\text { Expt } \\
\text { Ref. [2] }\end{array}$} \\
\hline & $\mathrm{a}$ & $\mathrm{c}$ & & & \\
\hline LFPO & 2.72 & 4.19 & 3.50 & 3.47 & 3.43 \\
\hline LMPO & N/A & N/A & 3.95 & 4.01 & 4.13 \\
\hline
\end{tabular}

Table S2. The lattice parameters of anitferromagnetic (AFM) configurations and the energy differences $E_{\text {total }}(A F M)-E_{\text {total }}(F M)$, given in unit of meV/formula unit.

\begin{tabular}{llllll}
\hline \hline LFPO & & $\mathrm{a}(\AA)$ & $\mathrm{b}(\AA)$ & $\mathrm{c}(\AA)$ & $\begin{array}{l}\text { Energy } \\
\text { difference }\end{array}$ \\
& GGA+U & 10.4047 & 6.0594 & 4.7383 & -11.03 \\
$\mathrm{LiFe}_{0.75} \mathrm{Mn}_{0.25} \mathrm{PO}_{4}$ & Ref.[3] & 10.3377 & 6.0112 & 4.6950 & N/A \\
& GGA+U & 10.4486 & 6.0818 & 4.7499 & -11.28 \\
Graphene & Ref.[4] & 10.356 & 6.027 & 4.706 & N/A \\
& GGA+U & 2.4683 & N/A & N/A & N/A \\
& Ref.[5] & 2.46 & N/A & N/A & N/A \\
\hline \hline
\end{tabular}

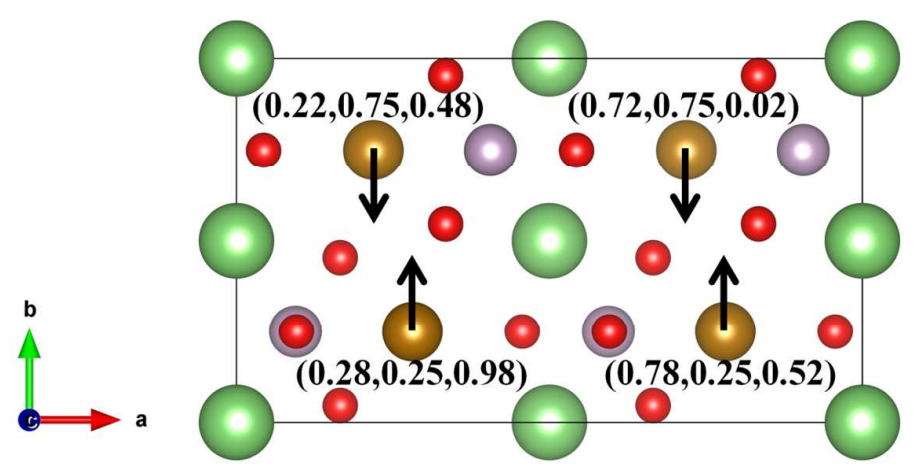

Figure S1. The AFM spin configuration for bulk LFPO. The green, brown, plum, red and circles represent $\mathrm{Li}, \mathrm{Fe}, \mathrm{P}$, and $\mathrm{O}$ atoms, respectively. The $\mathrm{Fe}$ atoms only along the $\mathrm{b}$ direction are antiparallelly arranged sequentially. The AFM spin configuration for $\mathrm{LiFe}_{0.25} \mathrm{Mn}_{0.75} \mathrm{PO}_{4}$ is same with that for LFPO. 
(a)

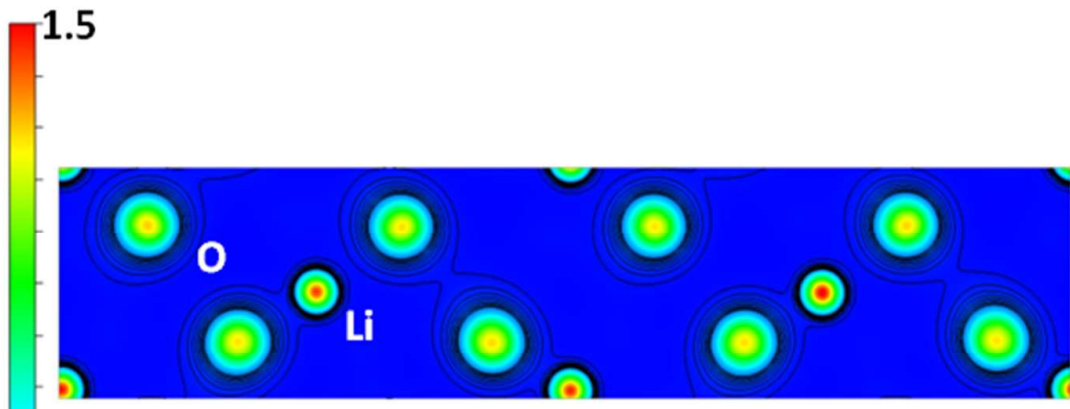

(b)

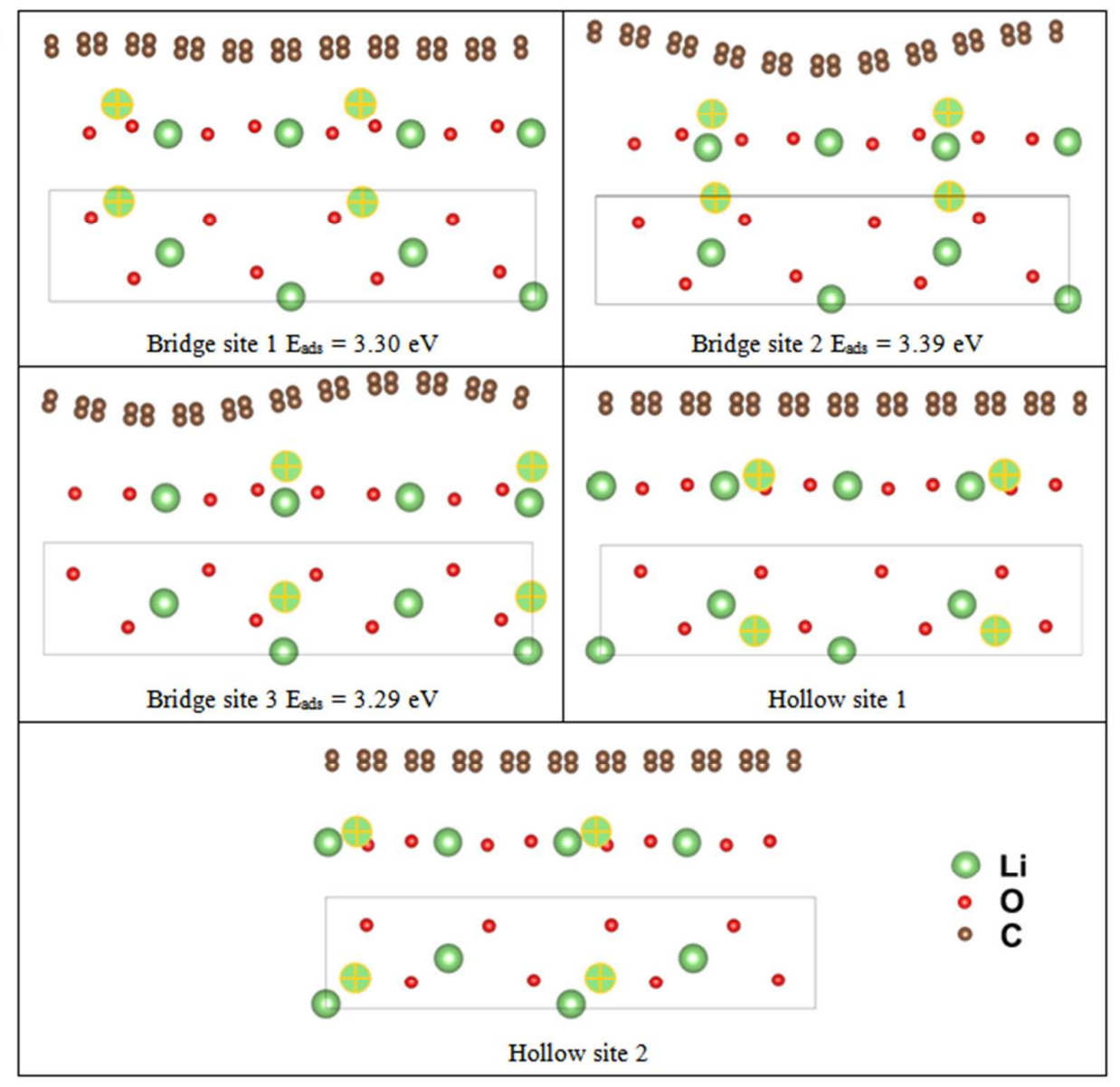

Figure S2. (a) Charge density contour of graphene/LFPO (G/LFPO) interface along b direction near the outermost $\mathrm{O}$ and $\mathrm{Li}$ atoms, where possible $\mathrm{Li}$ adsorption sites are selected at the area with low charge density, according to a previous study ${ }^{5}$. (b) Top and side views of other possible Li adsorption configurations on G/LFPO interface after geometry optimization and corresponding adsorption energy $E_{\text {ads }}$ except for hollow sites due to the relaxation of adatoms from hollow sites to bridge sites in the process of geometry optimization. Li atoms marked with yellow cross are adatoms. 
Table S3. The electron transfer (e) between graphene and olivine phosphate surfaces and the charge variation of different atoms in the process of interfacial binding based on the Bader analysis. Delithiated structures denote those after the delithiation of the outermost $\mathrm{Li}$ atoms, G/LFMPO denotes the graphene/Mn doped LFPO interface, outermost $\mathrm{O}$ denotes the sum of charge variation at 8 outermost $\mathrm{O}$ atoms, $\mathrm{O}_{1}$ denotes the $\mathrm{O}$ atom shown in Figure 3 (b), and TM and inner $\mathrm{O}$ denotes the sum of charge variation that is larger than $0.02 \mathrm{e}$ and occurs at $\mathrm{Fe}, \mathrm{Mn}$ and inner $\mathrm{O}$ atoms.

\begin{tabular}{lcccc}
\hline \hline G/LFPO & G/LFMPO & $\begin{array}{c}\text { Delithiated } \\
\text { G/LFPO }\end{array}$ & $\begin{array}{c}\text { Delithiated } \\
\text { G/LFMPO }\end{array}$ \\
\hline Graphene & -0.601 & -0.586 & -1.034 & -1.004 \\
Olivine phosphate & 0.600 & 0.587 & 1.034 & 1.003 \\
Outermost $\mathrm{O}$ & 0.463 & 0.451 & 0.126 & 0.009 \\
$\mathrm{O}_{1}$ & $\mathrm{~N} / \mathrm{A}$ & 0.018 & $\mathrm{~N} / \mathrm{A}$ & 0.033 \\
$\mathrm{TM}$ and inner O & 0.089 & 0.025 & 0.856 & 0.892 \\
\hline \hline
\end{tabular}

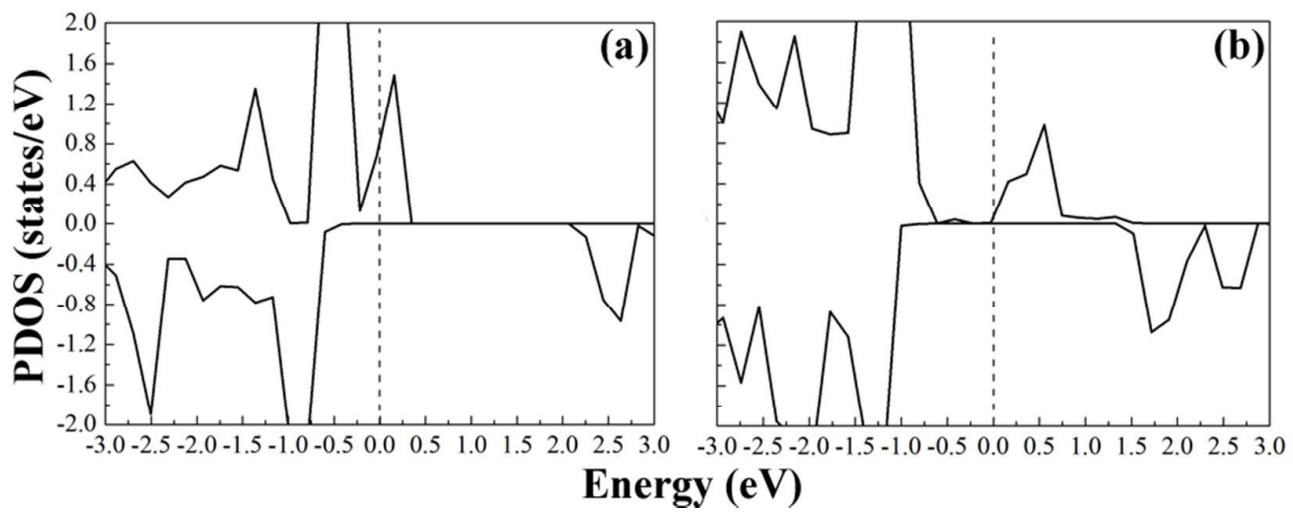

Figure S3. Spin-polarized density of states (DOS) projected on O-2p in the outermost layer of O atoms binding with (a) the adsorbed $\mathrm{Li}$ atoms at the LFPO surface, (b) the outermost $\mathrm{Li}$ atoms in the LFPO surface after the dilithiation of outermost Li atoms. The Fermi level is set as zero. 

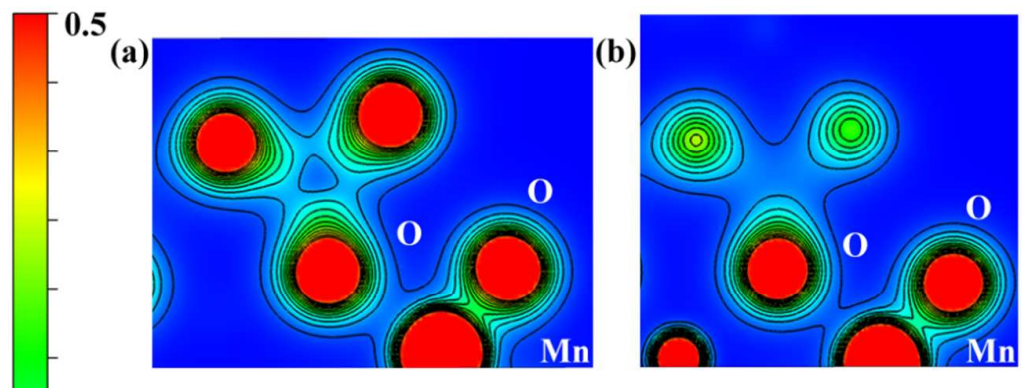

(c)

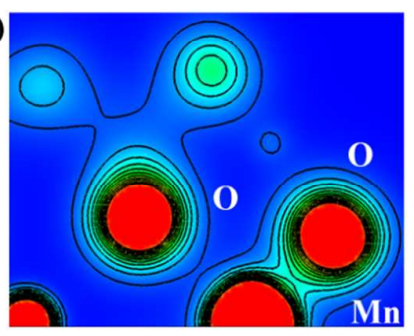

Figure S4. Charge density contours of Mn-O bonds in (a) LFMPO surface after the delithiation of the outermost Li atoms, (b) original LFMPO surface and (c) Li adsorbed LFMPO surface.

Table S4. The graphene maximum corrugation $(\AA)$ and graphene energies $(\mathrm{eV})$ relative to that in G/LFMPO interface. Delithiated G/LFPO and delithiated G/LFMPO denote the G/LFPO and G/LFMPO interfaces after the delithiation of the outermost Li atoms, respectively.

\begin{tabular}{lllll}
\hline \hline & G/LFPO & G/LFMPO & $\begin{array}{l}\text { Delithiated } \\
\text { G/LFPO }\end{array}$ & $\begin{array}{l}\text { Delithiated } \\
\text { G/LFMPO }\end{array}$ \\
\hline Maximum corrugation & 0.616 & 0.569 & 0.798 & 0.809 \\
Relative energy & 0.04 & 0.00 & 0.66 & 0.69 \\
\hline \hline
\end{tabular}

Table S5. The minimal distances $(\AA)$ between $\mathrm{Li}$ adatoms and $\mathrm{O}$ atoms and between $\mathrm{Li}$ adatoms and $\mathrm{C}$ atoms, the electron transfer (e) from two $\mathrm{Li}$ adatoms to olivine phosphate surface and to graphene in Li adsorbed G/LFPO and Li adsorbed G/LFMPO interfaces. Reference values of Li-O bond length in the bulk $\mathrm{Li}_{2} \mathrm{O}$ and of $\mathrm{Li}-\mathrm{C}$ bond length during the $\mathrm{Li}$ adsorption at the most stable site on graphene are listed for comparison.

\begin{tabular}{lllll}
\hline \hline & G/LFPO & G/LFMPO & & Reference value \\
\hline Li-O & 1.788 & 1.781 & Ref. [6] & 1.963 \\
Li-C & 2.347 & 2.300 & Ref. [7] & 2.24 \\
Olivine phosphate & 1.125 & 1.140 & & N/A \\
Graphene & 0.644 & 0.629 & & N/A \\
\hline \hline
\end{tabular}


The simulation and analysis of parallel binding between graphene and iron based olivine phosphates aims at probing the effect of local parallel structure of the graphene/LFPO interface observed in experiments ${ }^{8-12}$ on the electrochemical properties of the systems, and not necessarily relates to the hypothesis that the LFPO particle is entirely coated by pristine graphene so that the only way for $\mathrm{Li}$ ion to diffuse into and out of such particle may be the unlikely diffusion through the pristine graphene ${ }^{13}$. Besides the parallel binding between graphene and LFPO surface, the defect of graphene ${ }^{8}$, impurity of graphene ${ }^{10}$ and the perpendicular binding ${ }^{12}$ between the graphene and LFPO surface may appear at the interface, leading to the complexity of Li diffusion from electrolyte to LFPO surface that is left for further investigation.

Here, the barriers of Li diffusion on the LFPO surface and within the G/LFPO and G/LFMPO interfaces are calculated so as to clarify the effect of graphene modification and the coexistence of Mn dopant and graphene modification on the $\mathrm{Li}$ diffusion kinetics on the LFPO surface. Since the spin-polarized nudged elastic band (NEB) calculations of present model including about 180 atoms are time-consuming and give rise to convergence difficulty, the diffusion path is determined according to the previous calculation of $\mathrm{Li}$ diffusion on the $\mathrm{FePO}_{4}(010)$ surface ${ }^{14}$ and five transition configurations are constructed by linear interpolation of $\mathrm{Li}$ ion coordinates along [100] direction. The models and computation details for the surface and interface diffusion calculations are unchanged except that one $\mathrm{Li}$ ion vacancy is introduced at the final equilibrium configuration and that the $\mathrm{Li}$ ion chosen to diffuse is fixed along a axis, i.e. [100] direction, during the geometry optimization.

The optimized Li diffusion pathway and energy barriers for different systems are shown in Figure S5. The barrier of Li diffusion within the G/LFPO interface is 0.30 $\mathrm{eV}$ and is obviously lower than that $(0.62 \mathrm{eV})$ on the LFPO surface, for the transition configurations in G/LFPO interface is stabilized by the electrostactic attraction between the $\mathrm{Li}$ ion and neighboring $\mathrm{C}$ atoms. For example, while the interface binding of the initial configuration in G/LFPO interface belongs to the physical adsorption according to the discussion in 3.1 section, the distance between the $\mathrm{Li}$ ion and the nearest $\mathrm{C}$ atom of No. 3 configuration is $2.281 \AA$ which is even shorter than that between the Li adatom and the nearest $\mathrm{C}$ atom in Li adsorbed G/LFPO interface listed in Table S5. Since ionic bonds form between $\mathrm{Li}$ adatoms and graphene in $\mathrm{Li}$ adsorbed G/LFPO interface according to the discussion in 3.2 section, the same ionic bonds impossible for LFPO surface and initial configuration in G/LFPO interface are supposed to form for No. 3 transition configuration in G/LFPO interface, leading to the more stable transition configuration and the lower barrier of Li diffusion within G/LFPO interface.

On the other hand, the barrier of Li diffusion within the G/LFMPO interface is 0.40 $\mathrm{eV}$, obviously lower than that on the LFPO surface but mildly higher than that within the G/LFPO interface. The reason why the barrier of Li diffusion within the G/LFPO interface is elevated after the substitution of one outermost Fe by $\mathrm{Mn}$ is that the valence state of $\mathrm{Mn}$ is higher than that of $\mathrm{Fe}$ and the higher valence state of the metal ion nearest to the $\mathrm{Li}$ ion leads to the stronger electrostatic repulsion between them, thus destabilizing the transition configuration ${ }^{14-15}$. According to the Bader analysis for 
G/LFPO and G/LFMPO interfaces, the valence state of Mn is 1.739, higher than that (1.678) of the substituted Fe, consistent with the previous calculation ${ }^{4}$. Therefore, both the graphene modification and the coexistence of Mn dopant and graphene modification significantly improve the Li diffusion kinetics on the LFPO surface.
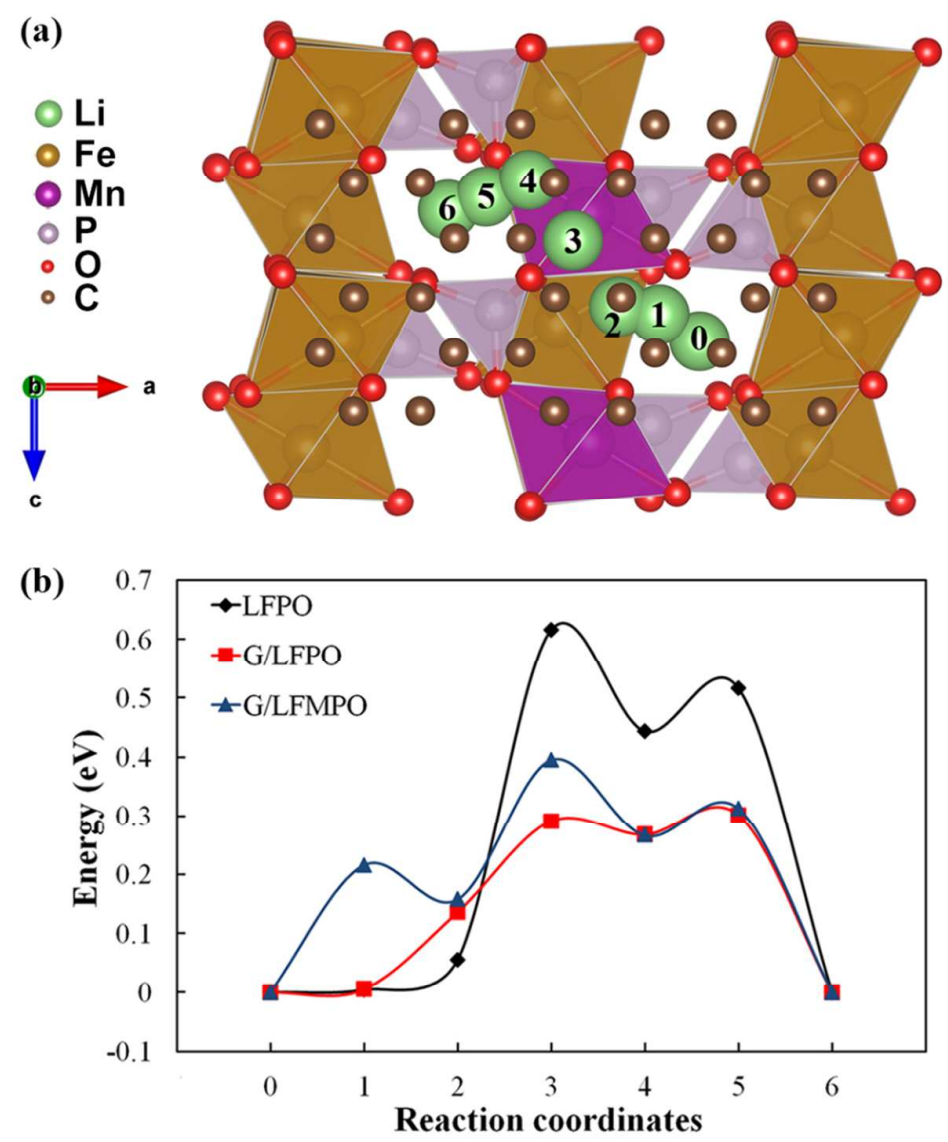

Figure S5. (a) Li diffusion pathway within the G/LFMPO interface. Pathways for LFPO surface and G/LFPO interface are similar, and all $\mathrm{Li}$ ions other than those on the pathway are removed for convenience. No. 0 and No. $6 \mathrm{Li}$ ions indicate the initial and final equilibrium configurations respectively, and No. 1 to No. $5 \mathrm{Li}$ ions indicate five transition configurations. (b) Energy barriers for Li diffusion on the LFPO surface, within the G/LFPO and G/LFMPO interfaces. The energies of equilibrium configurations are set to zero, respectively. 


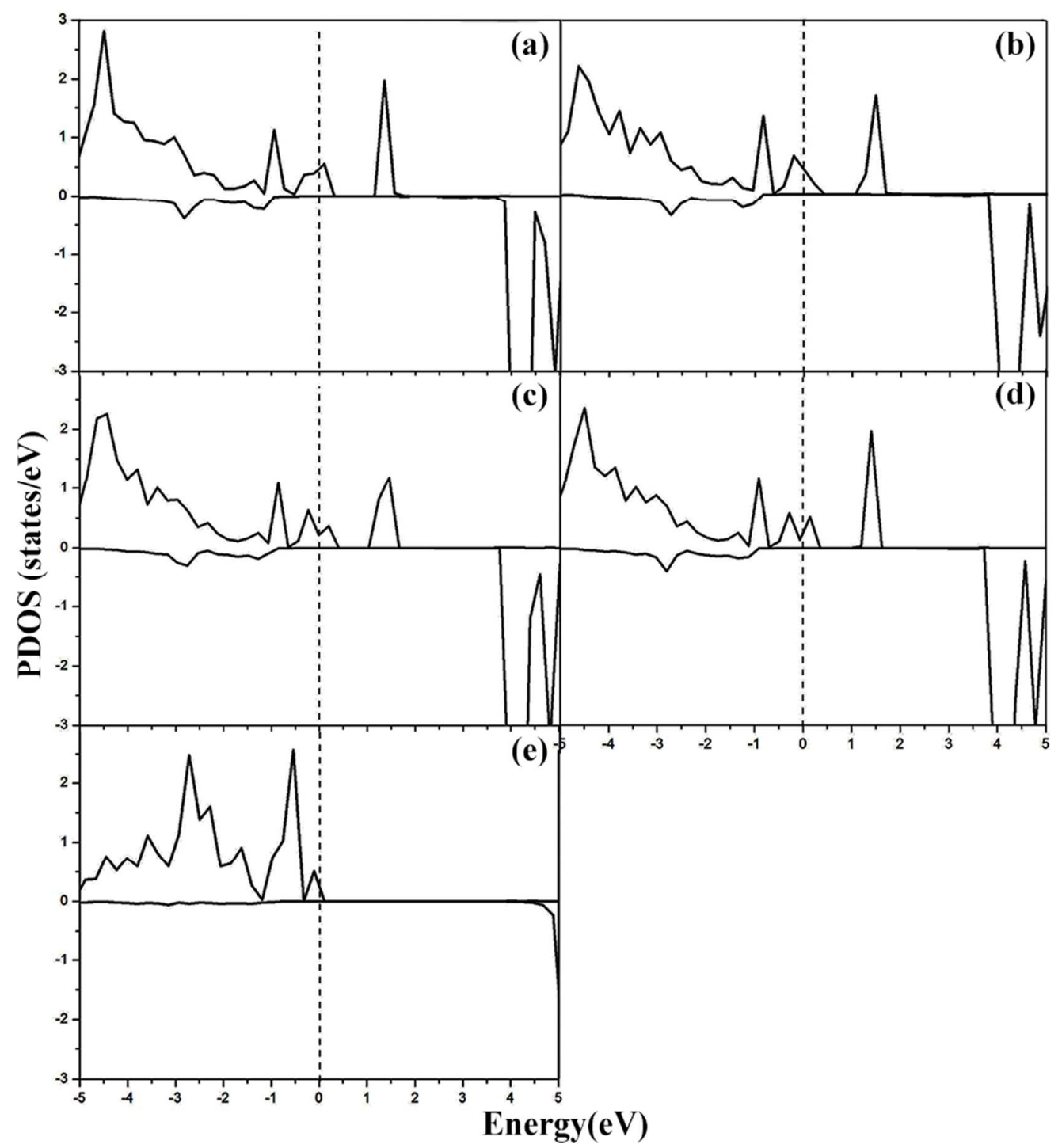

Figure S6. Spin-polarized DOS projected on Mn-3d in (a) G/LFMPO interface, (c) LFMPO surface and (e) LFMPO bulk. Counterparts after vdW correction are shown in (b) G/LFMPO interface and (d) LFMPO surface for comparison. The Fermi level is set as zero. 


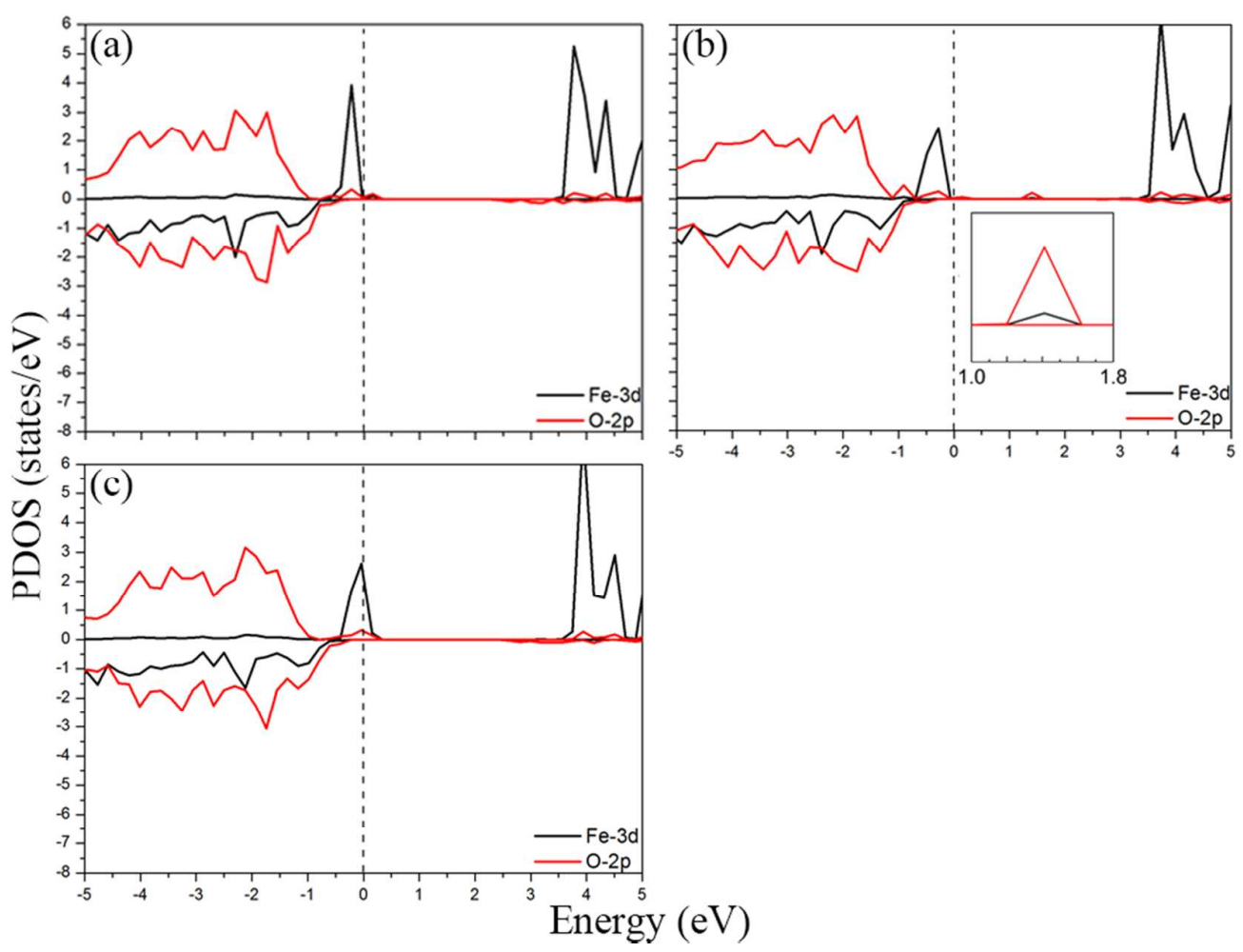

Figure S7. Spin-polarized local DOS projected on Fe-3d and O-2p in a $\mathrm{FeO}_{6}$ octahedron (a) in the second layer of LFPO surface, (b) nearest to the Mn dopant in the second layer of LFMPO surface and (c) in the second layer of G/LFPO interface. Inset in (c) shows projected DOS from 1.0 to 1.8 $\mathrm{eV}$. The Fermi level is set as zero.

1. Zhou, F.; Cococcioni, M.; Kang, K.; Ceder, G., The Li Intercalation Potential of Limpo4 and Limsio4 Olivines with M=Fe, Mn, Co, Ni. Electrochem. Commun. 2004, 6, 1144-1148.

2. Muraliganth, T.; Manthiram, A., Understanding the Shifts in the Redox Potentials of Olivine Lim1Y M Y Po4 (M= Fe, Mn, Co, and Mg) Solid Solution Cathodes. J. Phys. Chem. C 2010, 114, 15530-15540.

3. Rousse, G.; Rodriguez-Carvajal, J.; Patoux, S.; Masquelier, C., Magnetic Structures of the Triphylite Lifepo4 and of Its Delithiated Form Fepo4. Chem. Mater. 2003, 15, 4082-4090.

4. Osnis, A.; Kosa, M.; Aurbach, D.; Major, D. T., Systematic First-Principles Investigation of Mixed Transition Metal Olivine Phosphates Lim1-Ym' $\mathrm{Ypo}\left(\mathrm{M} / \mathrm{M}^{\prime}=\mathrm{Mn}, \mathrm{Fe}\right.$, and $\left.\mathrm{Co}\right)$ as Cathode Materials. J. Phys. Chem. C 2013, 117, 17919-17926.

5. Setiadi, J.; Arnold, M. D.; Ford, M. J., Li-Ion Adsorption and Diffusion on Two-Dimensional Silicon with Defects: A First Principles Study. ACS Appl Mater Interfaces 2013, 5, 10690-5.

6. Eithiraj, R. D.; Kalpana, G., Magnetism Induced by Nonmagnetic Dopant in Li2o, Na2o, K2o and Rb2o: First-Principles Calculations. Journal of Materials Science 2011, 47, 2316-2321.

7. Yildirim, H.; Kinaci, A.; Zhao, Z. J.; Chan, M. K.; Greeley, J. P., First-Principles Analysis of Defect-Mediated Li Adsorption on Graphene. ACS Appl Mater Interfaces 2014, 6, 21141-50.

8. Fei, H.; Peng, Z.; Yang, Y.; Li, L.; Raji, A. R.; Samuel, E. L.; Tour, J. M., Lifepo4 Nanoparticles Encapsulated in Graphene Nanoshells for High-Performance Lithium-Ion Battery Cathodes. Chem. Commun. 2014, 50, 7117-9. 
9. Lung-Hao Hu, B.; Wu, F. Y.; Lin, C. T.; Khlobystov, A. N.; Li, L. J., Graphene-Modified Lifepo(4) Cathode for Lithium Ion Battery Beyond Theoretical Capacity. Nat Commun 2013, 4, 1687.

10. Zhu, X.; Hu, J.; Wu, W.; Zeng, W.; Dai, H.; Du, Y.; Liu, Z.; Li, L.; Ji, H.; Zhu, Y., Lifepo4/Reduced Graphene Oxide Hybrid Cathode for Lithium Ion Battery with Outstanding Rate Performance. J. Mater. Chem. A 2014, 2, 7812.

11. Zhou, X.; Wang, F.; Zhu, Y.; Liu, Z., Graphene Modified Lifepo4 Cathode Materials for High Power Lithium Ion Batteries. J. Mater. Chem. 2011, 21, 3353.

12. Yang, J.; Wang, J.; Tang, Y.; Wang, D.; Li, X.; Hu, Y.; Li, R.; Liang, G.; Sham, T.-K.; Sun, X., Lifepo4-Graphene as a Superior Cathode Material for Rechargeable Lithium Batteries: Impact of Stacked Graphene and Unfolded Graphene. Energy Environ. Sci. 2013, 6, 1521.

13. Das, D.; Kim, S.; Lee, K. R.; Singh, A. K., Li Diffusion through Doped and Defected Graphene. PCCP 2013, 15, 15128-34.

14. Dathar, G. K. P.; Sheppard, D.; Stevenson, K. J.; Henkelman, G., Calculations of Li-Ion Diffusion in Olivine Phosphates. Chem. Mater. 2011, 23, 4032-4037.

15. Liu, Z.; Huang, X., Factors That Affect Activation Energy for Li Diffusion in Lifepo4: A First-Principles Investigation. Solid State Ionics 2010, 181, 907-913. 